\title{
Numerical Analysis of the Effects of Rotating Wind Turbine Blades on the Aerodynamic Forces Acting on Tower
}

\author{
Takaaki Kono $^{1, *}$, Satoshi Nebucho ${ }^{1}$, Tetsuya Kogaki ${ }^{2}$, Takahiro Kiwata ${ }^{1}$, Shigeo Kimura ${ }^{1}$ \\ and Nobuyoshi Komatsu ${ }^{1}$ \\ 1 Institute of Science and Engineering, Kanazawa University, Kanazawa 920-1192, Japan; \\ nebucho3104@gmail.com (S.N.); kiwata@se.kanazawa-u.ac.jp (T.K.); skimura@se.kanazawa-u.ac.jp (S.K.); \\ komatsu@se.kanazawa-u.ac.jp (N.K.) \\ 2 National Instiute of Advanced Industrial Science and Technology, Koriyama 963-0298, Japan; \\ kogaki.t@aist.go.jp \\ * Correspondence: t-kono@se.kanazawa-u.ac.jp; Tel.: +81-76-264-6469
}

Academic Editor: Lance Manuel

Received: 21 August 2016; Accepted: 13 January 2017; Published: 19 January 2017

\begin{abstract}
We have investigated the effects of the rotating blades of an upwind-type three-blade horizontal-axis wind turbine (HAWT) on the basic characteristics of aerodynamic forces acting on its tower by conducting improved delayed detached-eddy simulations (DESs). Three tip-speed ratios were considered for the operating conditions of the HAWT: $\lambda=3$ (low), $\lambda=6$ (optimum), and $\lambda=10$ (high). The diversion of the flow approaching the tower by the rotating blades and the low-pressure region that formed downwind of the blades significantly affected the aerodynamic forces acting on the tower. For example, the azimuth angle around the tower at which the pressure reached a maximum at each height shifted significantly in the direction of the movement of the blade passing the tower because of the diversion of the flow by the blades. Fluctuations in the lift force of the tower were significantly larger than those in its drag force because of the low-pressure region downwind of the blades.
\end{abstract}

Keywords: horizontal-axis wind turbine (HAWT); computational fluid dynamics (CFD); detached-eddy simulation (DES); tower; aerodynamic force; blade-tower interaction (BTI)

\section{Introduction}

When designing a wind turbine, it is important to avoid the excitation of resonant oscillations of the wind turbine tower by rotor thrust fluctuations at the blade-passing frequency or, to a lesser extent, at the rotational frequency. Wind turbine towers are customarily divided into three categories according to their stiffness: stiff, soft, and soft-soft [1-3]. A stiff tower is one whose fundamental natural frequency is above the blade-passing frequency range, a soft tower is one whose natural frequency is between the blade-passing frequency range and the rotor frequency range, and a soft-soft tower is one whose natural frequency is below both the rotor frequency range and the blade-passing frequency range. Because stiff towers usually require extra material not otherwise required for strength, soft towers are generally preferred. Soft and soft-soft towers can however be excited during the startup or shutdown of the turbine. In addition, dynamic magnification directly impacts the fatigue loads. Therefore, it is essential to design wind turbines and towers with the proper fatigue strength by considering their vibratory characteristics.

One of the main causes of rotor-thrust fluctuations at the blade-passing frequency is blade-tower interaction (BTI). Regardless of whether the configuration is upwind or downwind, the thrust force on the rotor of a horizontal-axis wind turbine (HAWT) decreases when a blade passes by its tower 
because the wind velocity of the wind approaching the tower and the wind velocity in the wake of the tower are reduced owing to the existence of the tower. The effects of the BTI on the rotor thrust have been extensively studied with computational fluid dynamics (CFD) simulations for upwind-type HAWTs [4-11] and downwind-type HAWTs [12,13]. However, studies on the effects of the BTI on the aerodynamic forces acting on the tower are very limited [11]. These studies involved CFD simulations of the National Renewable Energy Laboratory (NREL) Phase IV turbine [14] using Reynolds-averaged Navier-Stokes (RANS) turbulence models. Their main findings are as follows: Gómez-Iradi et al. [5] showed that the pressure on the upwind surface of the tower was reduced above the lowest point of the rotor at a certain rotational speed. This effect was evident even when the tips of the two blades were the maximum distance away from the tower (i.e., the angle between the tower and the blade was $90^{\circ}$ ). Wang et al. [9] reported that the pressure drop on the upwind surface of the tower, which Gómez-Iradi et al. [5] showed, was very weak at a certain tip-speed ratio when the tips of the two blades were relatively far from the tower. Using the HAWT with a downwind configuration, Zahle et al. [13] showed that the rotor had a strong effect on the tower shedding frequency, causing vortex lock-in at the upper part of the tower under certain flow conditions. They suggested the possibility that these lock-in phenomena were due to numerical artifacts of the RANS turbulence model. They also emphasized the necessity of further investigation using a large-eddy simulation (LES) or a hybrid RANS-LES model, which can reproduce separated flows more realistically.

In this study, as a first step toward clarifying the effects of aerodynamic forces acting on the tower of a wind turbine on the vibratory characteristics of the tower, we investigated the effects of the rotating blades of an upwind-type HAWT on the basic aerodynamic characteristics of the tower (e.g., the drag, lift, and pressure coefficients). This was done by conducting CFD simulations with the improved delayed detached-eddy simulation (IDDES) model [14], which is a hybrid RANS-LES model, of the wind flow around the wind turbine and its tower at low, optimum, and high tip-speed ratios.

\section{Numerical Approach}

In this study, a right-handed Cartesian coordinate system $\left(x_{1}, x_{2}, x_{3}\right)=(x, y, z)$ is employed, in which the $z$-direction is aligned in the vertical direction. The CFD software utilized to simulate the wind flow field is ANSYS Fluent 17.1 (ANSYS, Inc., Canonsburg, PA, USA) [15,16].

\subsection{Wind Turbine and Tower}

Figure 1 shows the geometry of the wind turbine and tower investigated by the CFD simulation. The rotor and nacelle are modeled as the upwind-type HAWT used by Eriksen and Krogstad [17] and Krogstad and Eriksen [18] in their wind tunnel experiments. The diameter of the rotor is $D=0.894 \mathrm{~m}$, and the center of the rotor is located $0.817 \mathrm{~m}$ above the floor. The blades have a non-uniform twist and chord-length distribution, and have an NREL S826 airfoil profile [19]. The tower has a uniform diameter of $d=0.061 \mathrm{~m}$ in the vertical direction. It is divided into 10 segments, and the aerodynamic forces acting on each segment are analyzed. The segments have identical lengths of $h=0.0772 \mathrm{~m}$.

\subsection{Governing Equations and Discretization Method}

The detached-eddy simulation (DES) model [20] is a hybrid RANS-LES model that treats near-wall regions in a RANS-like manner, and treats the rest of the flow in an LES-like manner. This model has the potential to save a large amount of computing resources compared with a pure LES model. However, modeled-stress depletion and grid-induced separation are problems in this model. The IDDES model mitigates these effects by delaying the RANS-to-LES transition and increasing the modeled stress contribution across the interface. 




(a)

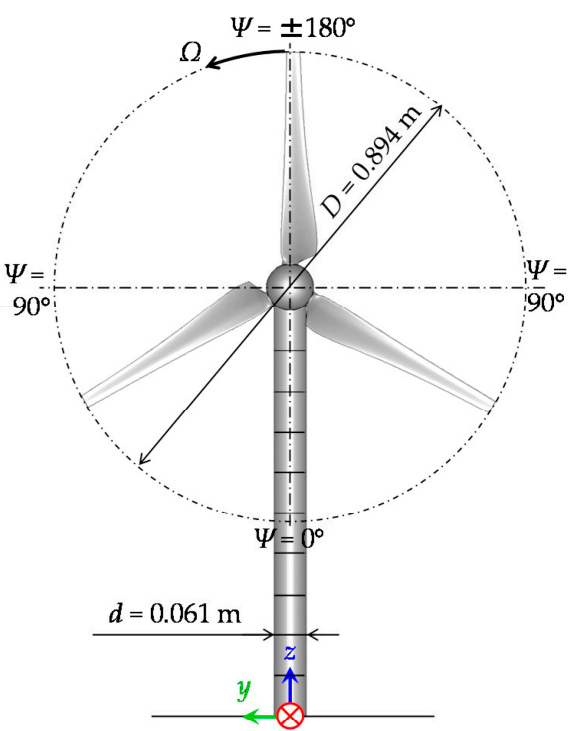

(b)

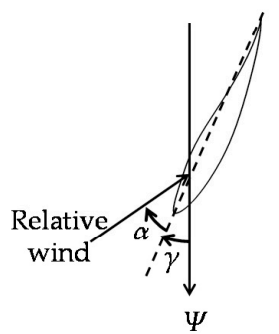

(c)

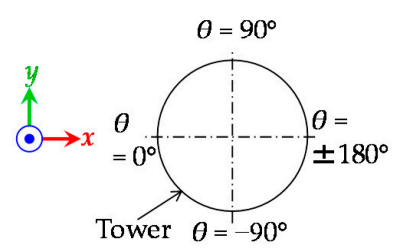

(d)

Figure 1. Geometry of the wind turbine and tower and the definitions of the angles $\Psi, \alpha, \gamma$, and $\theta$. (a) Side view of the wind turbine and tower; (b) front view of the wind turbine and tower; (c) cross-sectional view of the blade at $\Psi=0^{\circ}$; and (d) horizontal cross-sectional view of the tower.

The flow field around the wind turbine is assumed to be incompressible and isothermal in air at a temperature of $15{ }^{\circ} \mathrm{C}$. The governing equations for the IDDES model are the continuity equation:

$$
\frac{\partial u_{i}}{\partial x_{i}}=0
$$

the Navier-Stokes equation:

$$
\frac{\partial u_{i}}{\partial t}+\frac{\partial u_{i} u_{j}}{\partial x_{j}}=-\frac{1}{\rho} \frac{\partial p}{\partial x_{i}}+v \frac{\partial}{\partial x_{j}}\left(\frac{\partial u_{i}}{\partial x_{j}}+\frac{\partial u_{j}}{\partial x_{i}}-\frac{2}{3} \delta_{i j} \frac{\partial u_{i}}{\partial x_{j}}\right)-\frac{1}{\rho} \frac{\partial}{\partial x_{j}}\left[\mu_{t}\left(\frac{\partial u_{i}}{\partial x_{j}}+\frac{\partial u_{j}}{\partial x_{i}}\right)-\frac{2}{3} \rho k \delta_{i j}\right]
$$

the transport equation for the turbulence kinetic energy $k$ :

$$
\frac{\partial k}{\partial t}+\frac{\partial k u_{j}}{\partial x_{j}}=\frac{\partial}{\partial x_{j}}\left[\left(v+\frac{\mu_{t}}{\rho \sigma_{k}}\right) \frac{\partial k}{\partial x_{j}}\right]+\frac{2 \mu_{t}}{\rho} S_{i j} S_{i j}-\frac{k^{1.5}}{L_{I D D E S}}
$$

and the transport equation for the specific dissipation rate $\omega$ :

$$
\frac{\partial \omega}{\partial t}+\frac{\partial \omega u_{j}}{\partial x_{j}}=\frac{\partial}{\partial x_{j}}\left[\left(v+\frac{\mu_{t}}{\rho \sigma_{\omega}}\right) \frac{\partial \omega}{\partial x_{j}}\right]+\left(1-F_{1}\right) \frac{2}{\sigma_{\omega} \omega} \frac{\partial k}{\partial x_{j}} \frac{\partial \omega}{\partial x_{j}}+\frac{2 \alpha \omega \mu_{t}}{\rho k} S_{i j} S_{i j}-\beta \omega^{2}
$$

where $u_{i}$ is the wind-velocity component in the $x_{i}$ direction; $p$ is the pressure; $v$ is the kinematic viscosity; $\mu_{t}$ is the turbulence viscosity; $t$ is the time; $\rho$ is the air density; $S_{i j}$ is the mean strain rate; $L_{I D D E S}$ is the IDDES length scale [14]; $F_{1}$ is a blending function [21]; $\sigma_{k}$ and $\sigma_{\omega}$ are the turbulent Prandtl numbers for $k$ and $\omega$, respectively; and $\beta$ is a model constant [22]. $\mu_{t}$ is computed as follows:

$$
\mu_{t}=\frac{\rho k}{\omega} \frac{1}{\max \left[\frac{1}{\alpha *}, \frac{\sqrt{2 S_{i j} S_{i j}} F_{2}}{\alpha_{1} \omega}\right]}
$$


where $\alpha^{*}$ is a low-Reynolds number correction coefficient [21], $F_{2}$ is a blending function [21], and $\alpha_{1}$ is a model constant.

The governing equations are discretized by the finite-volume method. The advection terms of the Navier-Stokes equations are discretized by the bounded central-difference scheme. The advection terms of the transportation equations for $k$ and $\omega$ are discretized by the QUICK scheme. Other spatial derivatives are discretized by the central-difference scheme. The time integration is performed using the first-order implicit method.

\subsection{Numerical Setup}

The computational grids, the boundary conditions, and the total number of computational cells for all run cases are shown in Figure 2 and Table 1. The origin of the coordinate system is defined as the intersection point between the floor and the vertical line intersecting the center of the rotor. The computational domain consists of the rotational domain, which includes the rotor, and the non-rotating exterior domain. The size of the non-rotating exterior domain is $12.5 D \times 3 D \times 2 D$, which is approximately the same as that of the wind tunnel used by Krogstad and Eriksen in their experiments $[17,18]$. The diameters of the rotational domain $D_{R}$ for all run cases are listed in Table 1 . Here, $d_{t r}(=(1.13 D-D) / 2)$ is the shortest distance between the tip of a blade and the edge of the rotational domain. The blades, tower, and wind-tunnel walls are covered with boundary-layer meshes. Ideally, the first grid nodes over the surface of the blades should be set as $y+<1$ to resolve the eddies in the semi-viscous near-wall region. However, this requires an extremely large number of computational cells, in particular at a high tip-speed ratio.

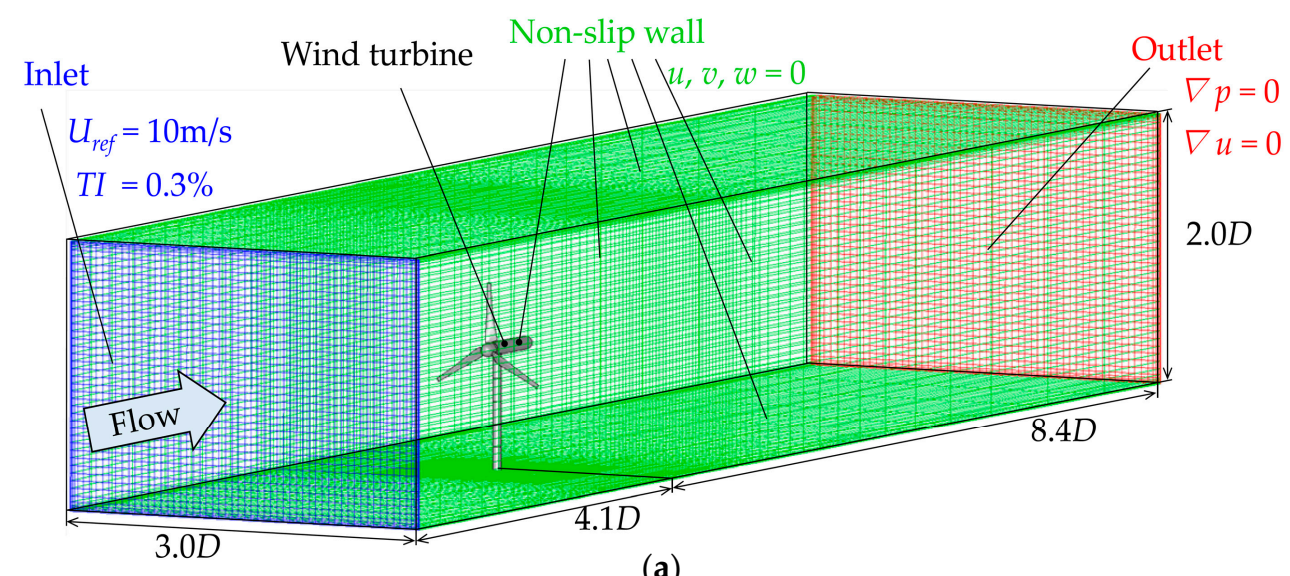

(a)

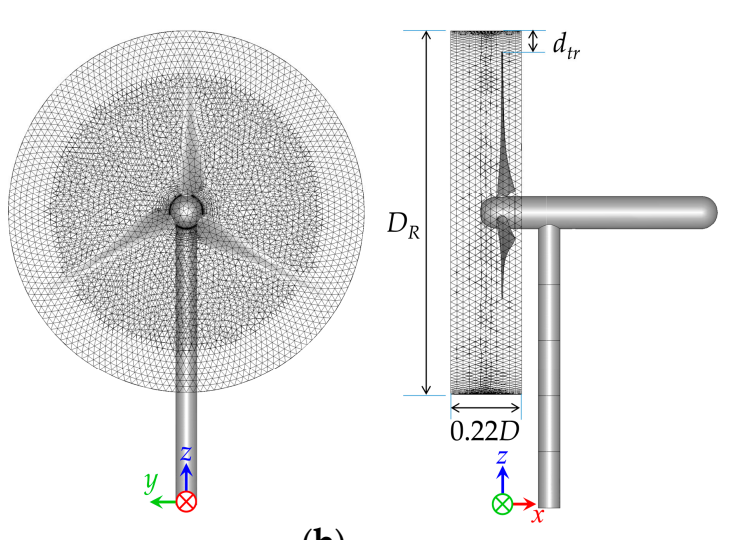

(b)



(c)

Figure 2. Computational grid and boundary conditions. (a) Entire computational domain; (b) rotational domain; and (c) horizontal cross-section of the grid near the tower at the mid-height of Segment 3. 
Table 1. Computational conditions. Here, $\lambda$ is the tip-speed ratio; $D_{R}$ is the diameter of the rotational domain; $D$ is the diameter of the rotor; $d_{t r}$ is the shortest distance between the tip of a blade and the edge of the rotational domain; and $d t$ is the time-step size.

\begin{tabular}{cccccc}
\hline Run Case & $\lambda$ & $\boldsymbol{D}_{\boldsymbol{R}}(\mathbf{m})$ & $\begin{array}{c}\text { Number of Cells in Structured Mesh } \\
\text { Region Surrounding the Tower }\end{array}$ & $\begin{array}{c}\text { Total Number } \\
\text { of Cells }\end{array}$ & $\boldsymbol{d} \boldsymbol{t}(\mathbf{s})$ \\
\hline$\lambda \_3$ & 3 & $1.13 D$ & $1,603,440$ & $8,568,292$ & $5.20 \times 10^{-4}$ \\
$\lambda \_6$ & 6 & $1.13 D$ & $1,603,440$ & $8,568,292$ & $2.60 \times 10^{-4}$ \\
$\lambda \_10$ & 10 & $1.13 D$ & $1,603,440$ & $8,568,292$ & $1.56 \times 10^{-4}$ \\
\hline DR_long & 6 & $1.13 D+2 d_{t r}$ & $1,603,440$ & $8,478,398$ & $2.60 \times 10^{-4}$ \\
D _short & 6 & $1.13 D-d_{t r}$ & $1,603,440$ & $8,705,489$ & $2.60 \times 10^{-4}$ \\
\hline G_fine & 6 & $1.13 D$ & $5,236,110$ & $12,805,094$ & $2.60 \times 10^{-4}$ \\
G_coarse & 6 & $1.13 D$ & 462,256 & $4,418,367$ & $2.60 \times 10^{-4}$ \\
\hline dt_fine & 6 & $1.13 D$ & $1,603,440$ & $8,568,292$ & $1.30 \times 10^{-4}$ \\
dt_coarse & 6 & $1.13 D$ & $1,603,440$ & $8,568,292$ & $5.20 \times 10^{-4}$ \\
\hline
\end{tabular}

According to grid-sensitivity studies on the NREL S826 airfoil, Krogstad and Lund [23] set the first grid nodes over the surface of the blades to be $y+<5$ in their CFD simulations for the same HAWT rotor that is used in the present study. They utilized the sear stress transfer (SST) $k-\omega$ turbulence model with low-Reynolds number corrections [21], which is the same turbulence model used in this study in the near-wall region. Because their CFD results for the power coefficients and thrust coefficients well matched their experimental results over a wide range of tip-speed ratios, we also set the first grid nodes over the surface of the blades to be $y+<5$ in all run cases by setting them as $2 \times 10^{-5} \mathrm{~m}$ off the surface of the blades.

The first grid nodes over the surface of the tower are also set at $2 \times 10^{-5} \mathrm{~m}$ off the surface of the tower, resulting in $y+<1$. As shown in Figure 2c, structured meshes are used in a region around and downwind of the tower. This region extends from $z=0$ to $z=9.9 \mathrm{~h}$. The numbers of the cells in this region are listed in Table 1 for all run cases. Here, case G_fine has a number of cells that is approximately 1.5 times larger in each direction $(x, y$, and $z)$ for the rectangular mesh and in each radius, $\theta$, and $z$ for the O-type mesh than case $\lambda \_6$. In addition, case $\lambda \_6$ has a number of cells that is approximately 1.5 times larger each direction $(x, y$, and $z)$ for the rectangular mesh, and in each radius, $\theta$, and $z$ for the O-type mesh than case G_coarse.

At the inlet boundary, a stream-wise wind velocity of $U_{r e f}=10 \mathrm{~m} / \mathrm{s}$ with a turbulence intensity of $T I=0.3 \%$ is implemented. At the outlet boundary, the outflow boundary condition is imposed. On the surfaces of the blades and tower and on the wind-tunnel walls, the no-slip boundary conditions are set. The sliding mesh technique is used to couple the rotational domain and the stationary domain. The direction of the rotor rotation is counterclockwise when viewed from upwind (Figure 1b). By changing the rotational speed of the rotor $\Omega$, the tip-speed ratio $\lambda\left(=D \Omega / 2 U_{r e f}\right)$ is set at 3,6 , or 10 . Except for cases dt_fine and dt_coarse, the time-step sizes are calculated as $d t=2^{\circ} / \Omega$. This yields time-step values of $5.20 \times 10^{-4} \mathrm{~s}$ when $\lambda=3,2.60 \times 10^{-4} \mathrm{~s}$ when $\lambda=6$, and $1.56 \times 10^{-4} \mathrm{~s}$ when $\lambda=10$. The time-step sizes for cases dt_fine and dt_coarse in Table 1 are calculated as $d t=1^{\circ} / \Omega$ and $d t=4^{\circ} / \Omega$, respectively. The statistics of the flow are calculated over the time interval $t \approx 0.9-2.8 \mathrm{~s}$, which is approximately 328 in non-dimensional time based on $d$. This provides a sufficient interval for evaluating the flow statistics around a circular cylinder [24]. The time-averaged value of an arbitrary physical quantity $\varphi$ is indicated as $\bar{\varphi}$.

\section{Results and Discussion}

\subsection{Validation of Numerical Approach}

Figure 3 compares the values of the power coefficient $C_{P}\left(=2 Q \Omega / \rho A U_{r e f}{ }^{3}\right)$ and the thrust coefficient $C_{T}\left(=2 T / \rho A U_{r e f}^{2}\right)$ evaluated from the simulations to those obtained from the wind-tunnel experiments [18]. Here, $Q$ is the torque on the rotor, $A$ is the rotor swept area, and $T$ is the total thrust 
on the blades. The simulations provide a reasonable qualitative and quantitative approximation of the experimentally observed results for both $C_{P}$ and $C_{T}$. The discrepancies of $C_{P}$ and $C_{T}$ between case $\lambda \_6$ and other cases with $\lambda=6$ are very small and are at most 0.0067 (dt_coarse) and 0.0048 (G_coarse), respectively.

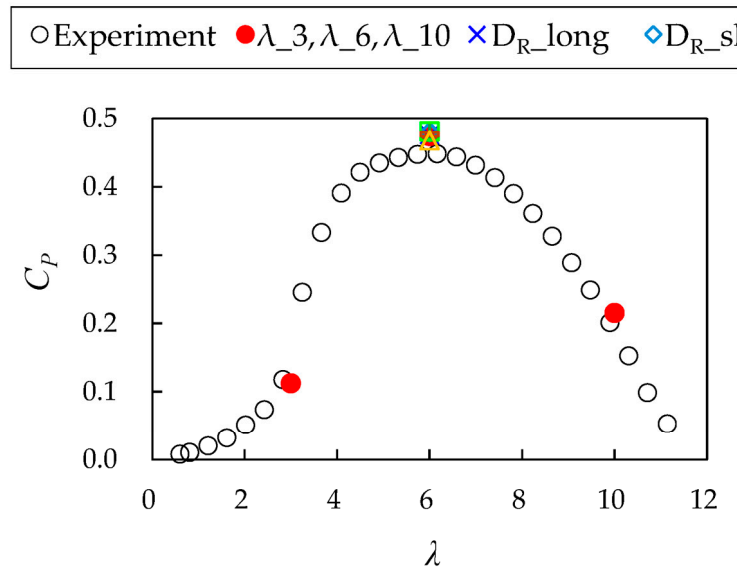

(a)

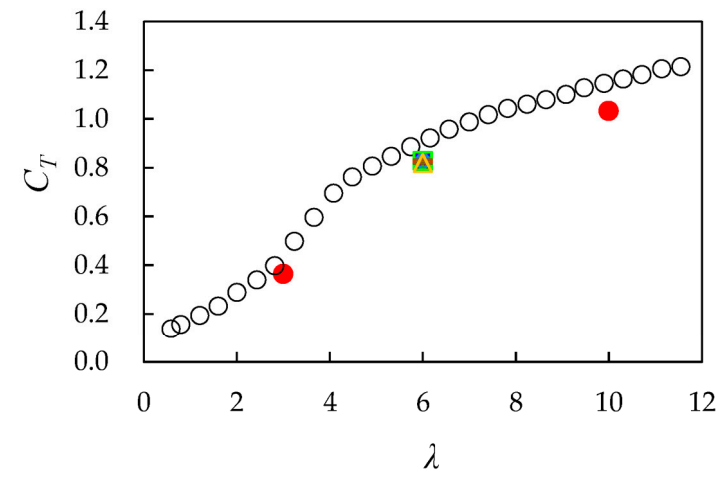

(b)

Figure 3. Computational and experimental results for the wind-turbine performance. (a) Power coefficient; and (b) thrust coefficient.

Figure 4 compares the simulation and wind-tunnel experiment results for the distribution in the $y$ direction of $\bar{u}$ at the hub height in the wind-turbine wake at $x / D=1$. The simulated values for $\bar{u}$ strongly match the experimental results $[17,18]$ qualitatively and quantitatively. The discrepancies of $\bar{u}$ between case $\lambda \_6$ and other cases with $\lambda=6$ are very small.

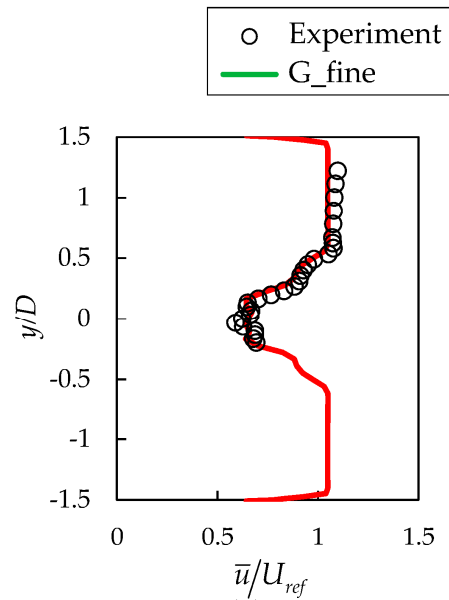

(a)

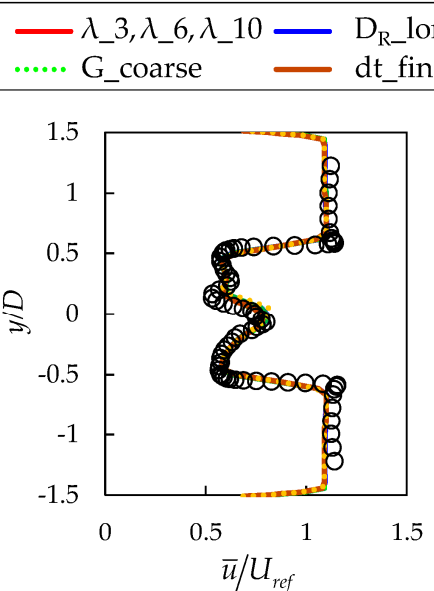

(b)

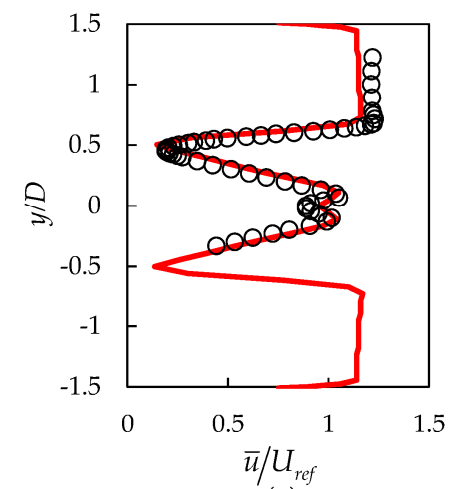

(c)

Figure 4. Lateral distribution of the streamwise wind velocity at the hub height in the wind-turbine wake at $x / D=1$. (a) $\lambda=3$; (b) $\lambda=6$; and (c) $\lambda=10$.

Figure 5 shows the sensitivity of $D_{R}$, the grid resolution of the structured mesh region near the tower, and $d t$ on the vertical distributions of the mean drag coefficient $C_{F x}\left(=2 \bar{F}_{x} / \rho h d U_{r e f}{ }^{2}\right)$, the mean lift coefficient $C_{F y}\left(=2 \bar{F}_{y} / \rho h d U_{r e f}{ }^{2}\right)$, the fluctuating drag coefficient $C_{F x}{ }^{\prime}\left(=2 \sigma_{F x} / \rho h d U_{r e f}{ }^{2}\right)$ and the fluctuating lift coefficient $C_{F y}{ }^{\prime}\left(=2 \sigma_{F y} / \rho h d U_{r e f}{ }^{2}\right)$ of each tower segment. Here, $F_{x}$ and $F_{y}$ are the drag and lift forces, respectively, acting on each tower segment, and $\sigma_{F x}$ and $\sigma_{F y}$ denote the root mean 
squares of $F_{x}$ and $F_{y}$, respectively. Except for case G_coarse, which is not shown in the figures, the discrepancies of these drag and lift coefficients between case $\lambda \_6$ and other cases with $\lambda=6$ are small.

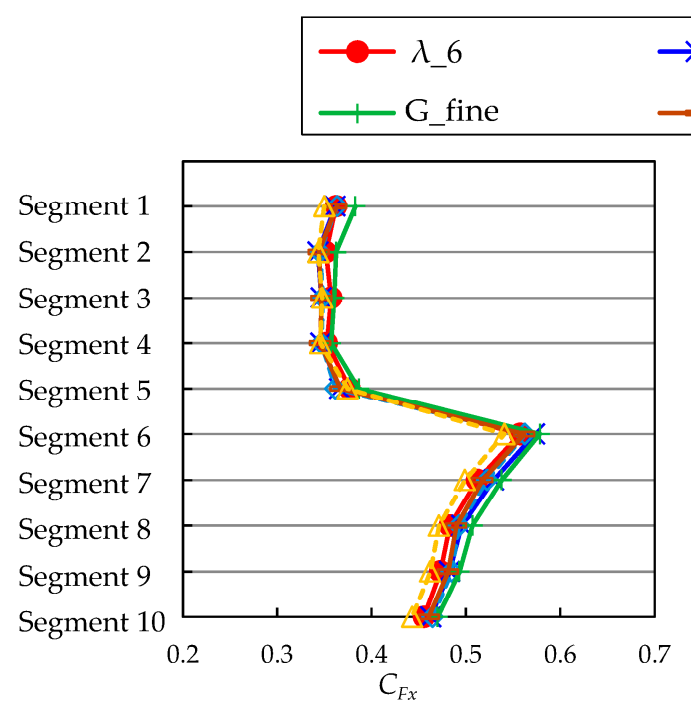

(a)

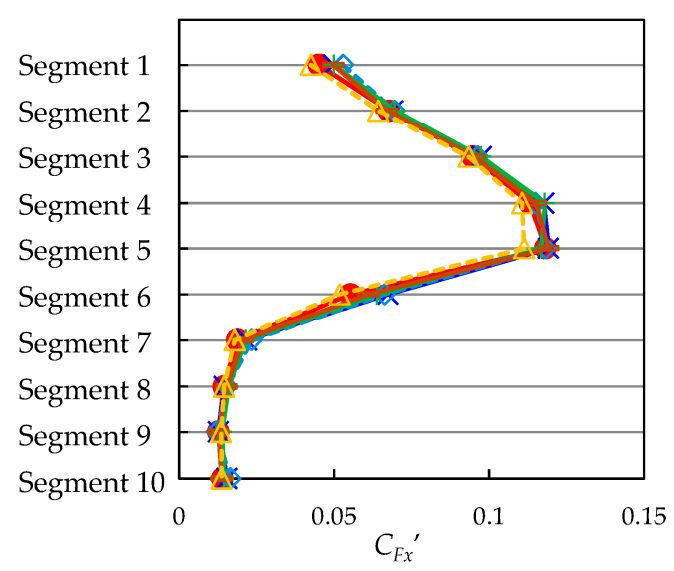

(c)
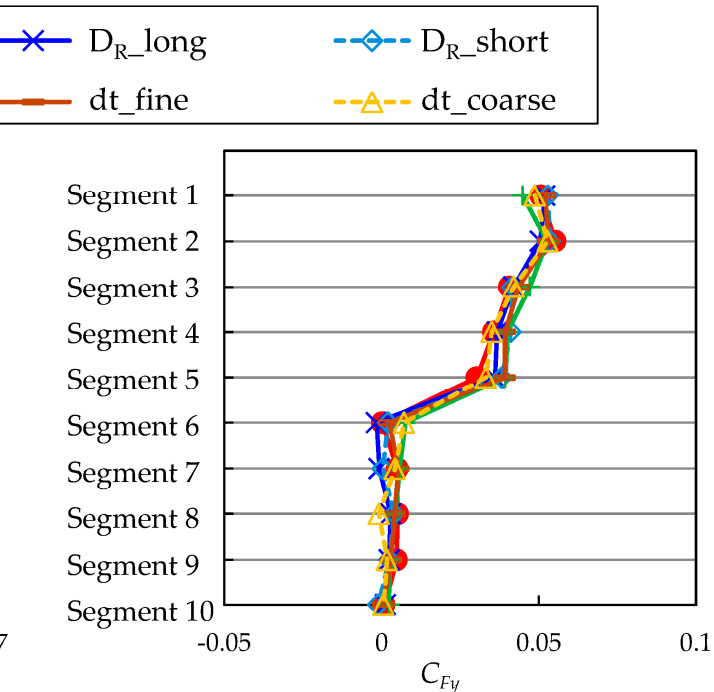

(b)

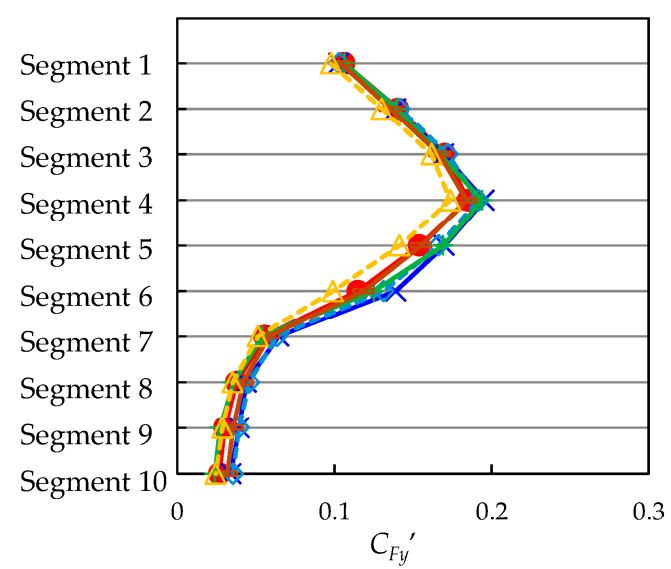

(d)

Figure 5. Sensitivity of $D_{R}$, the grid resolution near tower, and $d t$ on vertical distributions of the mean and fluctuating drag and lift coefficients of each tower segment at $\lambda=6$. (a) Mean drag coefficient; (b) mean lift coefficient; (c) fluctuating drag coefficient; and (d) fluctuating lift coefficient.

Figure 6 shows the sensitivity of $D_{R}$, the grid resolution of the structured mesh region near the tower, and $d t$ on the horizontal distributions of the mean pressure coefficient $C_{p}\left(=2\left(\bar{p}-p_{r e f}\right) / \rho U_{r e f}{ }^{2}\right)$ and the fluctuating pressure coefficient $C_{p}{ }^{\prime}\left(=2 \sigma_{p} / \rho U_{r e f}{ }^{2}\right)$ on the tower surface at the mid-height of Segment 6 . Here, $p_{\text {ref }}$ is the mean static pressure at $(-4.1 D, 0,0.91 D), \sigma_{p}$ denotes the root mean square of $p$, and $\theta$ is the azimuth angle around the tower, whose direction is defined in Figure $1 \mathrm{~d}$. Except for case G_coarse, which is not shown in the figures, the discrepancies of these pressure coefficients between case $\lambda \_6$ and other cases with $\lambda=6$ are small. This tendency is observed at other heights.

The aforementioned comparisons with the wind-tunnel experiments and the aforementioned sensitivity analyses indicate that the grid resolution, the rotational-domain size, and the time-step sizes for cases $\lambda \_3, \lambda \_6$, and $\lambda \_10$ are reasonable for discussing the characteristics of the aerodynamic forces acting on the tower. In the following sections, the results for cases $\lambda \_3, \lambda \_6$, and $\lambda \_10$ are discussed. 


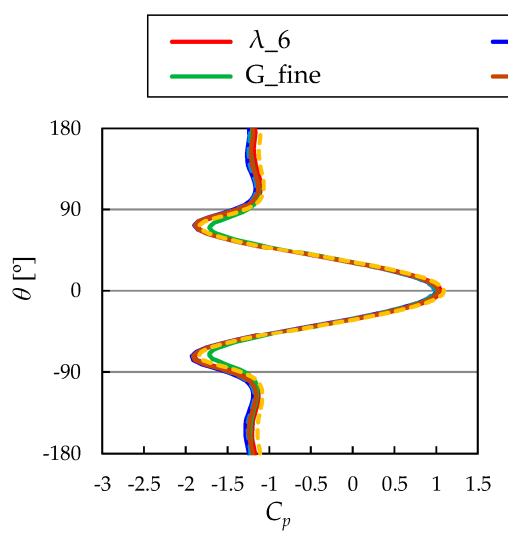

(a)

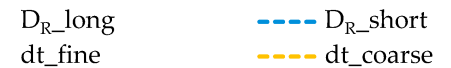

180

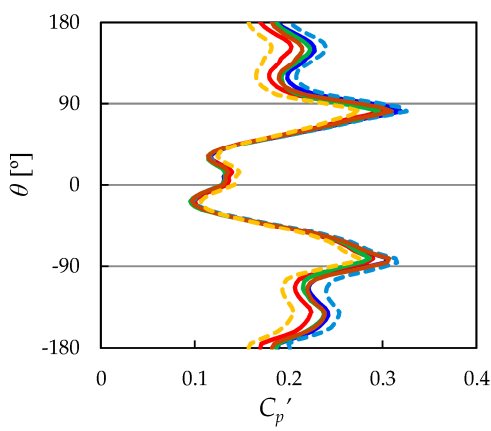

(b)

Figure 6. Sensitivity of $D_{R}$, the grid resolution around the tower, and $d t$ on distributions of the mean and fluctuating pressure coefficients on the tower surface at the mid-height of Segment 6 at $\lambda=6$.

(a) Mean pressure coefficient; and (b) fluctuating pressure coefficient.

\subsection{Pressure Coefficients on the Tower}

Figure 7 shows the distributions of $C_{p}$ on the tower surface at the mid-heights of each tower segment. When $\lambda=10$, the distributions of $C_{p}$ around the tower are almost symmetric with respect to $\theta=0$ for all segments. However, when $\lambda=3$ and $\lambda=6$, the angle $\theta$ at which $C_{p}$ is maximized shifts in the $-\theta$ direction at upper segments, particularly at segments 1 and 2 . This shifting is caused by the diversion of the flow approaching the tower by the rotating blades.

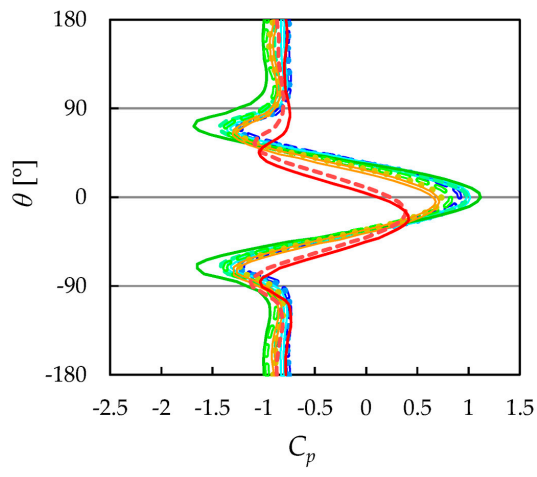

(a)



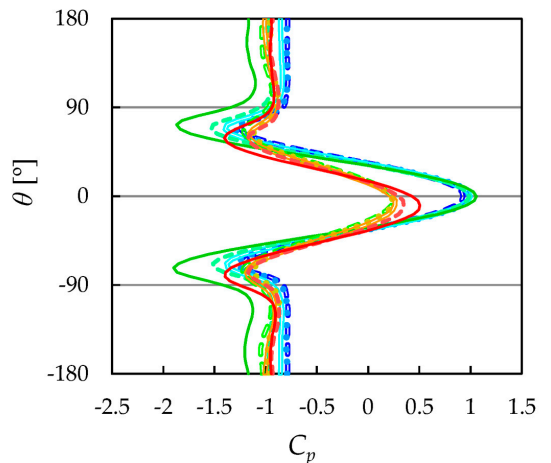

(b)

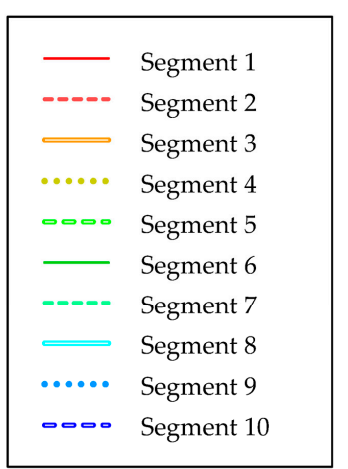

(c)

Figure 7. Distributions of the mean pressure coefficient on the tower surface at the mid-heights of each tower segment. (a) $\lambda=3$; (b) $\lambda=6$; and (c) $\lambda=10$. 
Figure $8 \mathrm{a}, \mathrm{b}$ show the streamlines at the mid-height of Segment 1 for $\lambda=3$ and $\lambda=6$, respectively. In both figures, at all values of $\Psi$, the blade near the tower significantly diverts the flow approaching the tower, and the stagnation point on the tower shifts in the $-\theta$ direction. The degree of the diversion of the flow is more significant when $\lambda=3$ than when $\lambda=6$. Conversely, when $\lambda=10$, the degree of the diversion of the flow due to the blade near the tower is very small at all values of $\Psi$, as shown in Figure 8c.

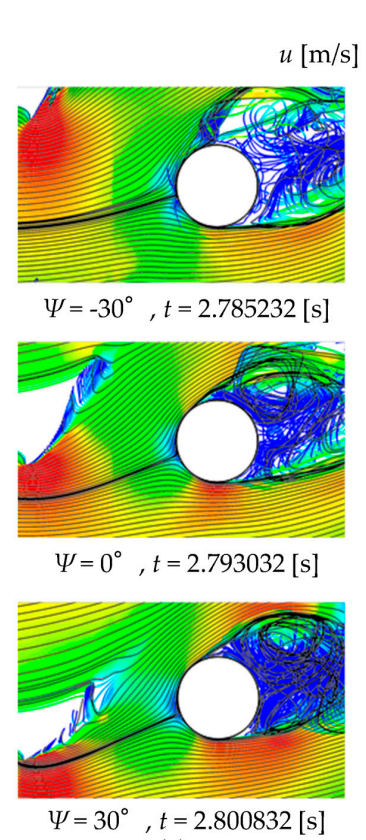

(a)

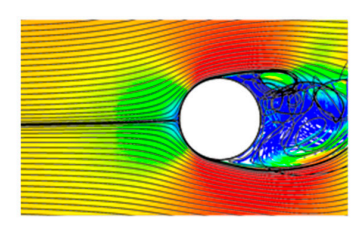

$\Psi=-30^{\circ}, t=2.785232[\mathrm{~s}]$

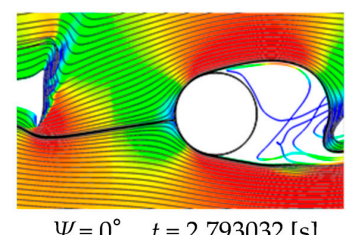

$\Psi=0^{\circ}, t=2.793032[\mathrm{~s}]$

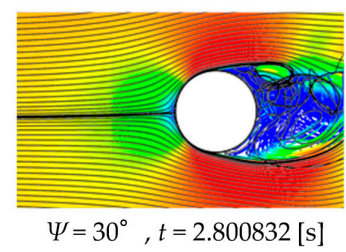

(d)

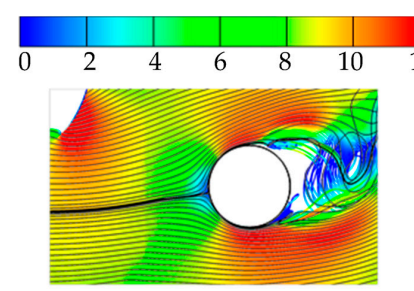

$\Psi=-30^{\circ}, t=2.796412[\mathrm{~s}]$

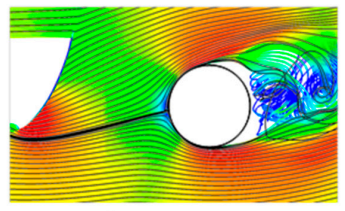

$\Psi=0^{\circ}, t=2.800312[\mathrm{~s}]$



(b)
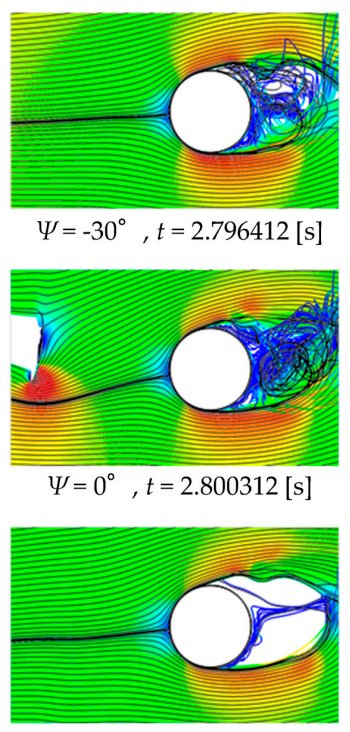

$\Psi=30^{\circ}, t=2.804212[\mathrm{~s}]$

(e)

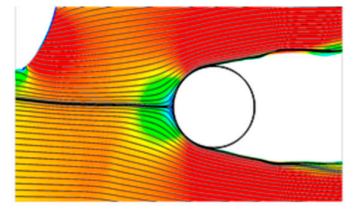

$\Psi=-30^{\circ}, t=2.792213[\mathrm{~s}]$

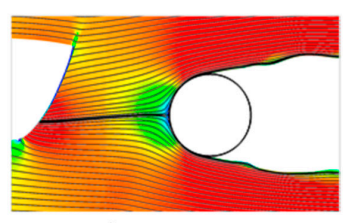

$\Psi=0^{\circ}, t=2.794554$ [s]

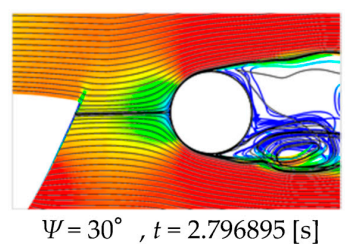

(c)

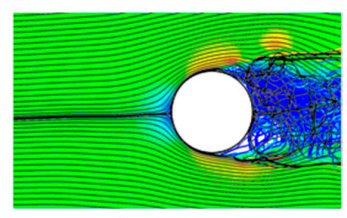

$\Psi=-30^{\circ}, t=2.792213[\mathrm{~s}]$

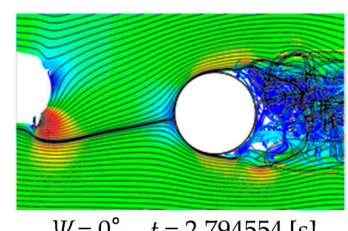

$\Psi=0^{\circ}, t=2.794554[\mathrm{~s}]$

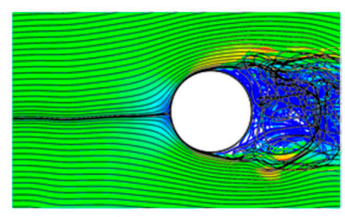

$\Psi=30^{\circ}, t=2.796895$ [s]

(f)

Figure 8. Instantaneous streamlines passing the line parallel to the $y$ axis and tangential to the tower on its upwind side at the mid-heights of Segments 1 and 4 . The circle indicates the cross-section of the tower. (a) $\lambda=3$, Segment 1 ; (b) $\lambda=6$, Segment 1 ; (c) $\lambda=10$, Segment 1 ; (d) $\lambda=3$, Segment 4 ; (e) $\lambda=6$, Segment 4 ; and (f) $\lambda=10$, Segment 4 .

Hence, the flow diversion caused by the rotating blades becomes less significant as $\lambda$ increases, which could be due to the decrease of the aerodynamic force acting on the blades in the $-\Psi$ direction 
arising from the reduction of the angle of attack on the blade ( $\alpha$, which is defined in Figure $1 c)$, as shown in Table 2. This leads to smaller drag and lift coefficients of the blades. Previous studies $[19,23]$ indicate that the lift coefficient of the S826 airfoil is 0 at $\alpha \approx-5^{\circ}$ and is maximized at $\alpha \approx 15^{\circ}$. Additionally, the drag coefficient of the S826 airfoil is in the range of approximately $0.5-1.0$ when $-10^{\circ} \leq \alpha \leq 15^{\circ}$ and increases sharply at $\alpha \geq 15^{\circ}$ owing to the onset of a stall. The decreases in $\alpha$ and the aerodynamic force acting on the blades with an increase in $\lambda$ leads to higher $u$ values in the region between the rotor and tower, as shown in Figure 8a-c; higher $p$ values on the downwind side of the blade, as shown in Figure 9; and higher maximum $C_{p}$ values around the tower at the mid-height of segment 1 , as shown in Figure 7.

Table 2. Angle of attack of the blade at $\Psi=0^{\circ}$ at the mid-height of each tower segment.

\begin{tabular}{cccc}
\hline Segment of the Tower & $\alpha$ When $\lambda=3$ & $\alpha$ When $\lambda=6$ & $\alpha$ When $\lambda=\mathbf{1 0}$ \\
\hline 1 & $28.59^{\circ}$ & $9.59^{\circ}$ & $-3.98^{\circ}$ \\
2 & $25.28^{\circ}$ & $7.32^{\circ}$ & $-2.01^{\circ}$ \\
3 & $21.74^{\circ}$ & $7.07^{\circ}$ & $0.33^{\circ}$ \\
4 & $19.16^{\circ}$ & $7.16^{\circ}$ & $1.93^{\circ}$ \\
5 & $17.75^{\circ}$ & $7.71^{\circ}$ & $3.46^{\circ}$ \\
\hline
\end{tabular}

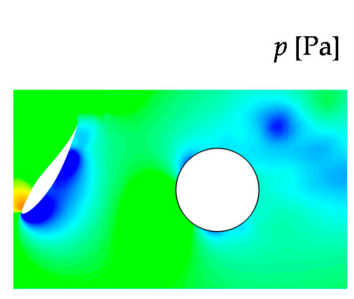

(a)

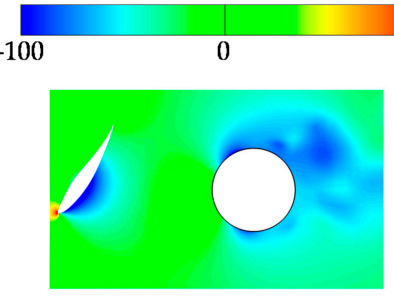

(b)

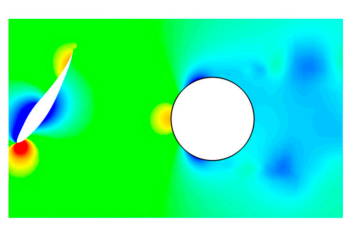

(c)

Figure 9. Horizontal distributions of the static pressure at the mid-height of segment 1 when the blade is located at $\Psi=0^{\circ}$. (a) $\lambda=3, t=2.793032$ (s); (b) $\lambda=6, t=2.800312$ (s); and (c) $\lambda=10, t=2.794554$ (s).

When $\lambda=3$, the maximum values of $C_{p}$ at the mid-heights of Segments 3-5 are larger than those of Segments 1 and 2, as shown in Figure 7a. This is mainly attributed to the decrease in the drag force acting on the blades, which reduces the wind velocity. The decrease in the drag force acting on the blade with an increase in the radial distance from the rotational axis (or with a decrease in the distance from the floor when the blade is at $\Psi=0^{\circ}$ ) is mainly attributed to the decrease in $\alpha$, as shown in Table 2 . When $\lambda=6$ and $\lambda=10$, the maximum values of $C_{p}$ at the mid-heights of Segments 3-5 are smaller than those of Segments 1 and 2, as shown in Figure $7 \mathrm{~b}, \mathrm{c}$, respectively. This tendency is more significant when $\lambda=10$, mainly because of the increase in the lift force acting on the blade, which reduces the wind velocity. The increase in the lift force acting on the blades with an increase in the radial distance from the rotational axis could be caused by the increase in the relative wind velocity experienced by the blade. When $\lambda=10$, the increase in the lift force acting on the blades with an increase in the radial distance from the rotational axis could also be a result of the increase in the lift coefficient of the blade with an increase in $\alpha$.

In Figure $8 \mathrm{~d}-\mathrm{f}$, the flow approaching the tower is significantly diverted by the blade when $\Psi=0^{\circ}$. However, for all other values of $\Psi$, the diversion of the flow approaching the tower by the blades is very small. This explains that the amount of shifting of $\theta$ at which $C_{p}$ is maximized is very small at the mid-heights of Segments 3-5 for all the $\lambda$ values shown in Figure 7.

Figure 10 shows the distributions of $C_{p}{ }^{\prime}$ on the tower surface at the mid-heights of each tower segment. For all $\lambda$ values, the distributions of $C_{p}{ }^{\prime}$ around the tower are almost symmetrical with respect to $\theta=0$ at the segments near the floor. However, with the exception of these segments, the values of $C_{p}{ }^{\prime}$ are generally higher in the $+\theta$ region than in the $-\theta$ region. This tendency could be 
caused by two factors when a blade passes the tower segments. The first factor is the low pressure region that forms on the downwind side of the blade. This region lowers the pressure on the tower. The second factor is the diversion of the flow approaching the tower by the blade, which shifts the stagnation point on the tower in the $-\theta$ direction.

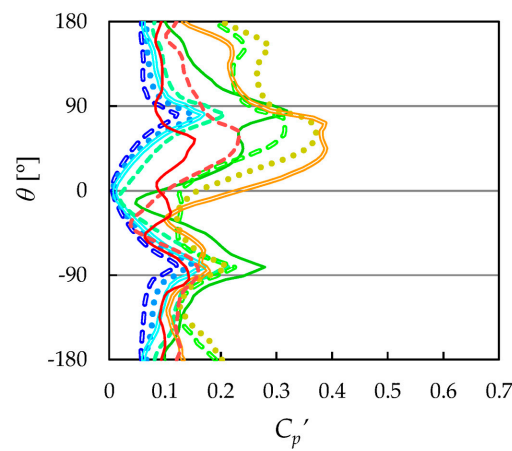

(a)

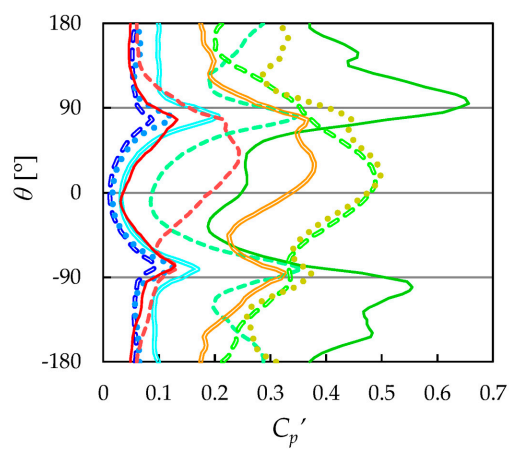

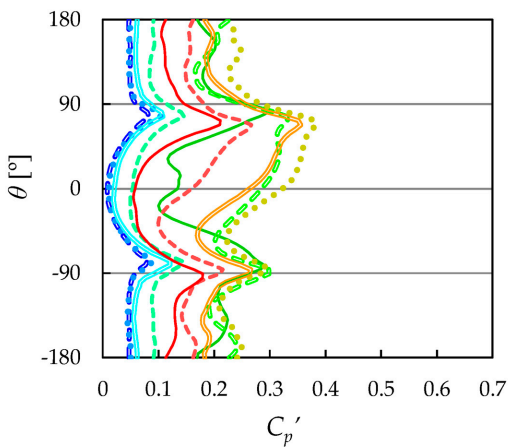

(b)

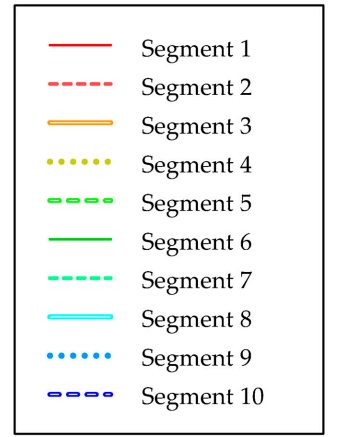

(c)

Figure 10. Distributions of the fluctuating pressure coefficient on the tower surface at the mid-heights of each tower segment. (a) $\lambda=3$; (b) $\lambda=6$; and (c) $\lambda=10$.

Figure 11 shows the pressure distribution at the mid-height of segment 4 . As indicated by the figure, the pressure near the tower is reduced for all $\lambda$ values as the blade approaches the tower. The pressure recovers when the blade moves away from the tower. Figure 12 shows the distributions of the instantaneous pressure coefficient $C_{p i}\left(=2\left(p-p_{r e f}\right) / \rho U_{r e f}{ }^{2}\right)$ on the tower surface at the mid-heights of segment 4 when the blade nearest the tower is at various $\Psi$ values. As shown in this figure, the $\theta$ at which $C_{p i}$ is maximized on the upwind side of the tower generally shifts in the $-\theta$ direction when the blade is near the tower (such as when $\Psi=-20^{\circ}$ to $20^{\circ}$ ) for all $\lambda$ values. This shift in $\theta$ at which $C_{p i}$ is maximized is due to the shifting of the stagnation point on the tower.

When the blade approaches the tower, the values of $C_{p i}$ on the $+\theta$ side are generally lower than those on the $-\theta$-side. This is because the distance from the lower-pressure region on the downwind side of the blade is shorter on the $+\theta$ side than that on the $-\theta$-side. Conversely, when the blade moves away from the tower, the values of $C_{p i}$ on the $-\theta$-side are generally lower than those on the $+\theta$ side. This is due to the distance from the lower-pressure region on the downwind side of the blade. However, when the blade moves away from the tower, the decrease in $p$ on the $-\theta$-side surface of the tower is suppressed by the shifting of $\theta$ at which $C_{p i}$ is maximized in the $-\theta$ direction owing to the shifting of the stagnation point. Therefore, the degree of decrease in $p$ on the $-\theta$-side surface of the tower when the blade moves away from the tower is generally smaller than that on the $+\theta$-side surface of the tower when the blade approaches the tower. As a result, the values of $C_{p}{ }^{\prime}$ are generally higher on the $+\theta$-side surface of the tower than those on the $-\theta$-side surface. 



(b)


$t=2.789872[\mathrm{~s}] \quad t=2.792993[\mathrm{~s}] \quad t=2.793774$ [s]

(c)

Figure 11. Horizontal distributions of the instantaneous static pressure at the mid-height of Segment 4 . (a) $\lambda=3$; (b) $\lambda=6$; and (c) $\lambda=10$.

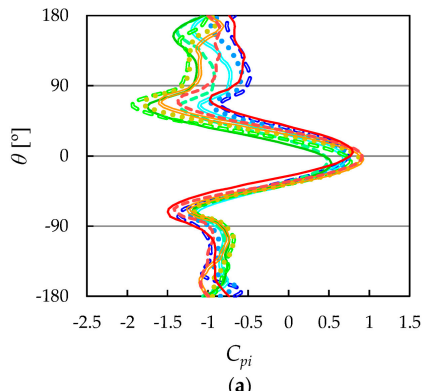

(a)

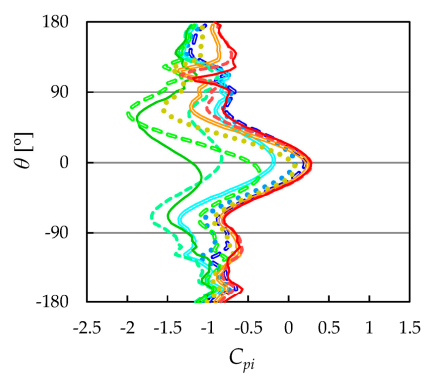

(c)

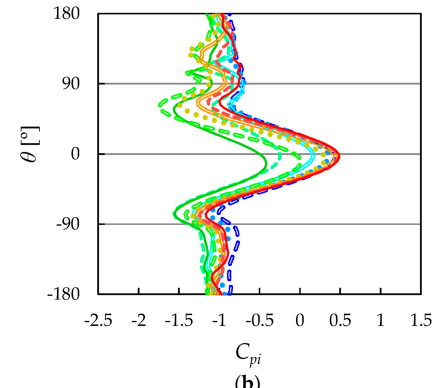

(b)

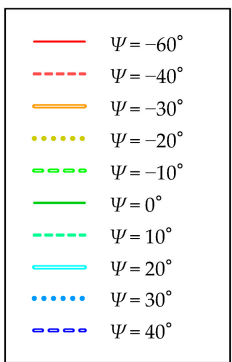

Figure 12. Distributions of the instantaneous pressure coefficient on the tower surface at the mid-height of Segment 4 for moments when the blade nearest the tower is at various $\Psi$ values. (a) $\lambda=3$; (b) $\lambda=6$; and $(\mathbf{c}) \lambda=10$. 


\subsection{Drag and Lift Coefficients of the Tower}

Figure 13 shows the vertical distributions of $C_{F x}$ and $C_{F y}$ of each tower segment. Figure 13a indicates that $C_{F x}$ is maximized at Segment 6 when $\lambda=3$ and $\lambda=6$, and at Segment 7 when $\lambda=10$, in Figure 13a. Hence, $C_{F x}$ is the maximum at a height close to and lower than the lowest point of the rotor for all values of $\lambda$. This is because at Segment 6 or 7 , the values of $\bar{p}$ are the lowest or second-lowest downwind of the tower, whereas the values of $\bar{p}$ upwind of the tower are approximately equal to those at the lower segments, as shown in Figure 7.

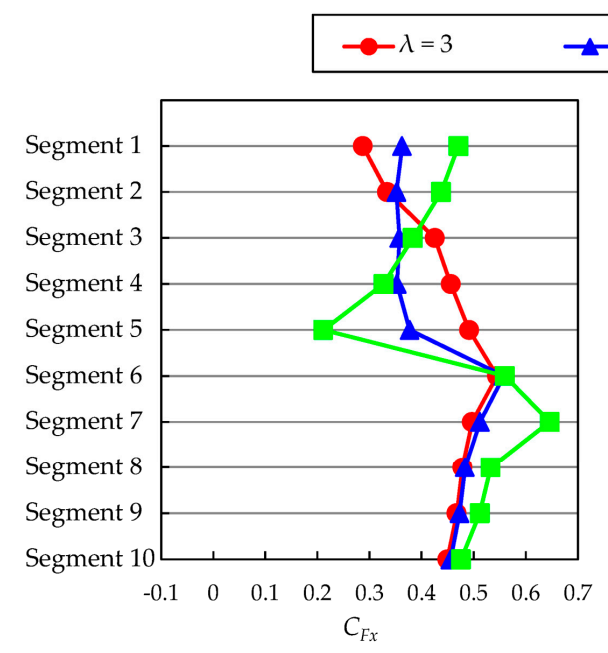

(a)

$$
\neg \lambda=6 \quad-\lambda=10
$$

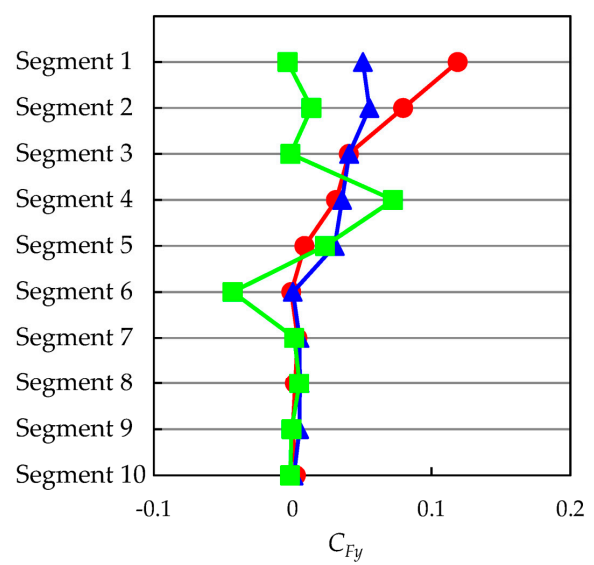

(b)

Figure 13. Vertical distributions of the mean drag and mean lift coefficients of each tower segment. (a) Mean drag coefficient; and (b) mean lift coefficient.

The aerodynamic force acting on the upper segments of the tower could be very important from the perspective of the overturning moment of the tower. In Figure 13a, the values of $C_{F x}$ at Segments 1 and 2 increase as $\lambda$ increases. This is because the values of $\bar{p}$ upwind of the tower increase as $\lambda$ increases as shown in Figure 7. The values of $C_{F y}$ shown in Figure $13 \mathrm{~b}$ are very small compared with those of $C_{F x}$. In Figure 13b, the values of $C_{F y}$ at Segments 1 and 2 increase as $\lambda$ decreases. This is mainly because the values of $\bar{p}$ on the $-\theta$-side increase owing to the increased shift in the stagnation point in the $-\theta$ direction as $\lambda$ decreases, as shown in Figure 7.

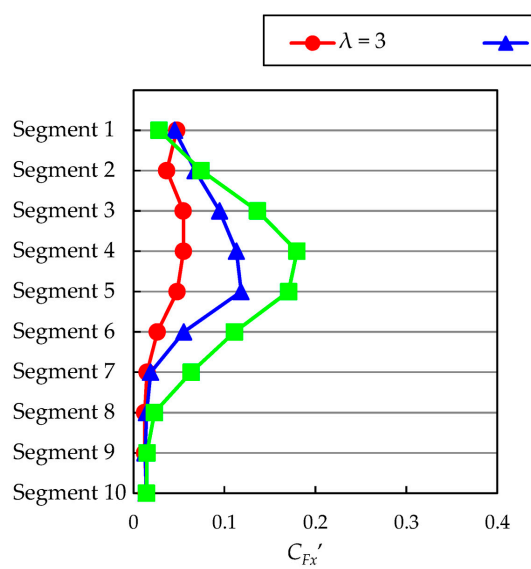

(a)

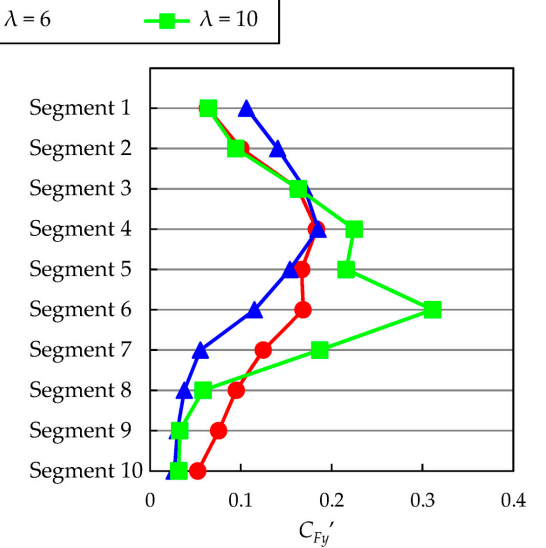

(b)

Figure 14. Vertical distributions of the fluctuating drag and fluctuating lift coefficients of each tower segment. (a) Fluctuating drag coefficient; and (b) fluctuating lift coefficient. 
Figure 14 shows the vertical distributions of $C_{F x}{ }^{\prime}$ and $C_{F y}{ }^{\prime}$ of each tower segment. Here, the values of $C_{F x}{ }^{\prime}$ are smaller than those of $C_{F y}{ }^{\prime}$ at all segments for all values of $\lambda$. Figure 14a shows that for all values of $\lambda$, the values of $C_{F x}{ }^{\prime}$ at Segments 1-5, which are higher than the lowest point of the rotor, are larger than the values of $C_{F x}{ }^{\prime}$ at the segments near the floor.

Additionally, the values of $C_{F x}{ }^{\prime}$ are particularly large at Segments 3-5 and are maximized at one of these segments for all values of $\lambda$. The large fluctuations in $F_{x}$ at these segments are caused by the decrease and subsequent increase in $F_{x}$ that occur when a blade approaches and then moves away from the tower, as shown in Figure 15. This figure shows the time-history curves for the instantaneous drag coefficient $C_{F x i}\left(=2 F_{x} / \rho h d U_{r e f}^{2}\right)$ of each tower segment.

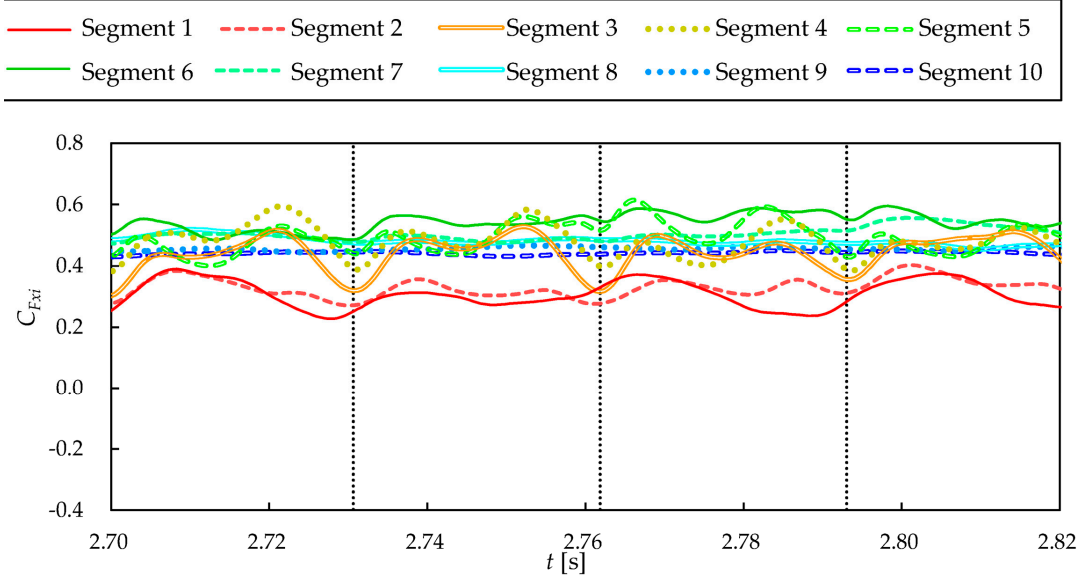

(a)

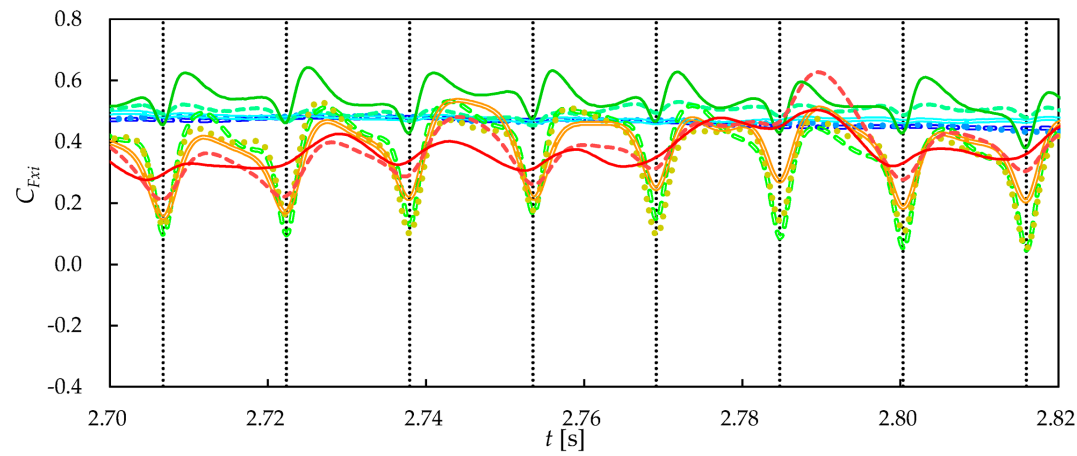

(b)

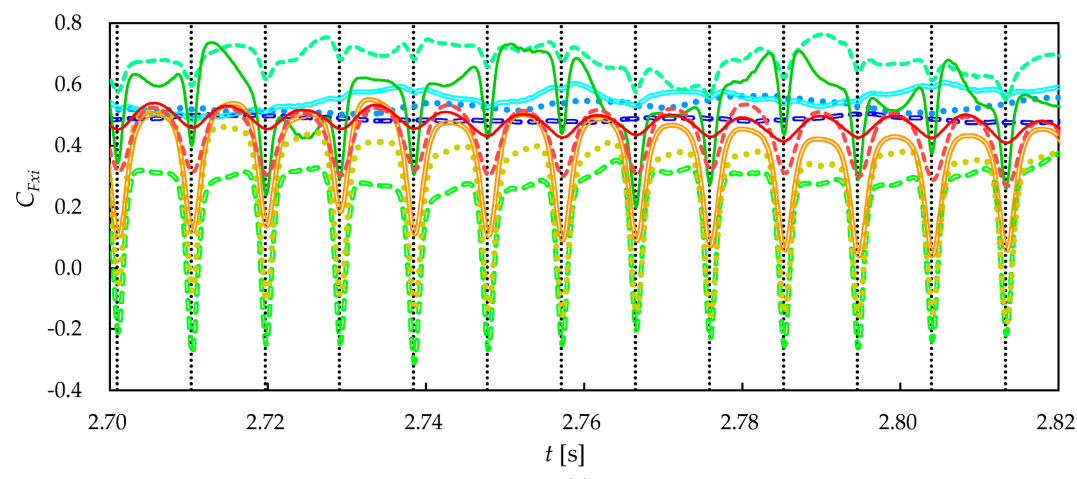

(c)

Figure 15. Time history of the instantaneous drag coefficient of each tower segment. The vertical dotted lines indicate the time at which the blade nearest the tower is at $\Psi=0^{\circ}$. (a) $\lambda=3$; (b) $\lambda=6$; and (c) $\lambda=10$. 
The decrease and subsequent increase in $F_{x}$ at Segments 3-5 that occur when a blade approaches and then moves away from the tower are mainly due to the decrease in the values of $p$ upwind of the tower segments caused by the lower-pressure region formed downwind of the blade, as discussed in the previous section. With an increase in $\lambda$, the magnitude of the decrease in $F_{x}$ increases, as shown in Figure 15, and the maximum values of $C_{F x}{ }^{\prime}$ at Segments 3-5 increase, as shown in Figure 14a, because the values of $p$ downwind of the blade decrease. The value of $C_{F x}{ }^{\prime}$ at Segment 1 in Figure 14a for $\lambda=10$ is smaller than that for $\lambda=3$ and $\lambda=6$. This is because when $\lambda=10$, the decrease in $p$ downwind of the blade that passes the tower is very small owing to the small $\alpha$. The values of $C_{F x}{ }^{\prime}$ at $\lambda=3$ and $\lambda=6$ are approximately equal. However, the fluctuations in $p$ are larger upwind of the tower when $\lambda=3$ and downwind of the tower when $\lambda=6$, as shown in Figure 10.

Figure 14b shows that like $C_{F x}{ }^{\prime}$, the values of $C_{F y}{ }^{\prime}$ at Segments 1-5 are larger than the values of $C_{F y}{ }^{\prime}$ at the segments near the floor for all values of $\lambda$. When $\lambda=3$ and $\lambda=6$, the values of $C_{F y}{ }^{\prime}$ are maximized at Segment 4 . When $\lambda=10$, the value of $C_{F y}{ }^{\prime}$ at segment 4 is the second-largest. At Segment 6, which is lower than the lowest point of the rotor, the value of $C_{F y}{ }^{\prime}$ is maximized. The values of $C_{F y}{ }^{\prime}$ are relatively large at Segments 3-5 for all values of $\lambda$ because when a blade approaches the tower, $F_{y}$ increases and then decreases, and when the blade moves away from the tower, $F_{y}$ decreases further and then increases, as shown in Figure 16. Figure 16 shows the time history of the instantaneous lift coefficient $C_{F y i}\left(=2 F_{y} / \rho h d U_{\text {ref }}{ }^{2}\right)$ of each tower segment. Periodic fluctuation in $F_{y}$ occurs because the low-pressure region downwind of the blade has a greater effect on the pressure on the $+\theta$-side surface of the tower segments when the blade approaches the tower and a greater effect on the pressure on the $-\theta$-side surface of the tower segments when the blade moves away from the tower, as shown in Figure 11.

$C_{F y}{ }^{\prime}$ is maximized at Segment 6 when $\lambda=10$ because in addition to the aforementioned fluctuation in $F_{y}$ that occurs when a blade passes the tower, the $F_{y}$ values fluctuate with the largest amplitude and smaller frequency than the blade-passing frequency, as shown in Figure 16. In this figure, $F_{y}$ is negative at $t \approx 2.72-2.77 \mathrm{~s}$ and positive at $t \approx 2.77-2.81 \mathrm{~s}$, with a large amplitude. This is because a strong vortex tends to form on one lateral side of segment 6 for longer periods than the blade-passing interval, followed by a similar formation on the other lateral side. Figure $14 \mathrm{~b}$ shows that at segment 1 , $C_{F y}{ }^{\prime}$ is larger for $\lambda=6$ than the values obtained when $\lambda=3$ and $\lambda=10$. This is because the value of $p$ on the tower segment in the vicinity of $\theta= \pm 90^{\circ}$ fluctuates more when $\lambda=6$, as shown in Figure 10.

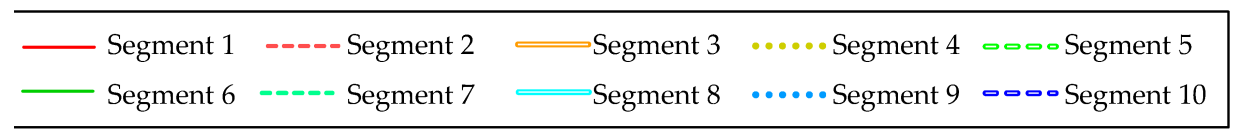



(a)

Figure 16. Cont. 


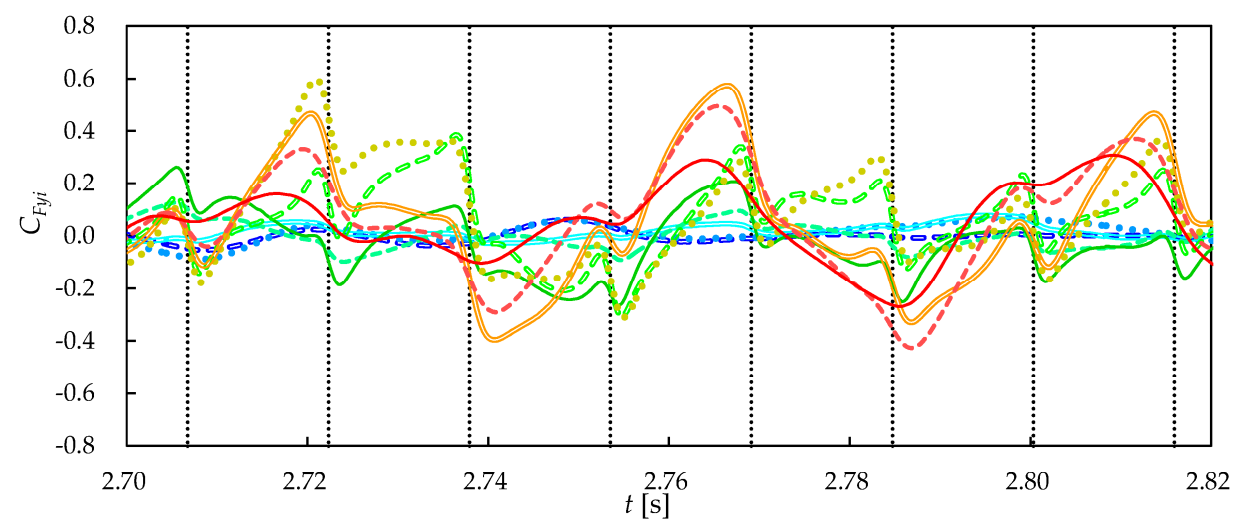

(b)



(c)

Figure 16. Time history of the instantaneous lift coefficient of each tower segment. The vertical dotted lines indicate the time at which the blade nearest the tower is at $\Psi=0^{\circ}$. (a) $\lambda=3$; (b) $\lambda=6$; and (c) $\lambda=10$.

\section{Conclusions}

As a first step toward clarifying the effects of aerodynamic forces acting on the tower of a wind turbine on the vibratory characteristics of the tower, we investigated the effects of the rotating blades of an upwind-type three-blade HAWT on the basic characteristics of the aerodynamic forces acting on its tower. This was done by conducting improved delayed detached-eddy simulations of the wind flow around the tower and the wind turbine operating at tip-speed ratios of $\lambda=3$ (low), $\lambda=6$ (optimum), and $\lambda=10$ (high). The tower was divided into 10 equal segments, and the aerodynamic forces acting on each segment were analyzed.

We discovered that both the diversion of the flow approaching the tower by the rotating blades and the low-pressure region that forms downwind of the blades have significant effects on the aerodynamic forces acting on the tower. For example, owing to the diversion of the flow by the blades, the azimuth angle around the tower at which the pressure reaches a maximum at each height can shift significantly in the direction of the movement of the blade passing the tower. In addition, because of the low-pressure region downwind of the blades, the fluctuation in the lift force of the tower can be significantly larger than that in its drag force.

These characteristics of the aerodynamic forces acting on the tower might contribute significantly to the occurrence of unacceptably large stresses and deflections in the side-to-side direction when the blade passing frequency coincides with the natural frequency of the tower. Owing to the negligible contribution of the aerodynamic damping of HAWTs in the side-to-side direction, the damping ratio for the side-to-side oscillations of the tower is generally one order of magnitude less than that for the fore-aft oscillations of the tower [2]. Therefore, in future research, we plan to investigate the effects of 
the aerodynamic forces acting on the tower that are influenced by the rotating blades on the vibratory characteristics of the tower in the side-to-side direction by conducting fluid-structure interaction simulations. We also plan to further investigate the effects of the rotating blades of the HAWT on the basic characteristics of the aerodynamic forces acting on the tower by varying the diameter of the tower, the distance between the rotor and the tower, etc.

Acknowledgments: This research was supported by New Energy and Industrial Technology Development Organization (Project No. P13010). We thank Per-Åge Krogstad of the Norwegian University of Science and Technology for providing us with the geometric data for the wind turbine.

Author Contributions: Takaaki Kono and Satoshi Nebucho performed the numerical simulations. Takaaki Kono and Satoshi Nebucho analyzed the data with supervision by Tetsuya Kogaki, Takahiro Kiwata, Shigeo Kimura and Nobuyosi Komatsu.

Conflicts of Interest: The authors declare no conflict of interest.

\section{References}

1. Manwell, J.F.; Mcgowan, J.G.; Rogers, A.L. Wind Energy Explained Theory, Design and Application, 2nd ed.; WILEY, John Wiely \& Sons Ltd.: Chichester, UK, 2009; p. 379.

2. Burton, T.; Jenkins, N.; Sharpe, D.; Bossanyi, E. Wind Energy Handbook, 2nd ed.; WILEY, John Wiely \& Sons Ltd.: Chichester, UK, 2011; pp. 303-305.

3. Licari, J.; Ekanayake, J.B.; Jenkins, N. Investigation of a speed exclusion zone to prevent tower resonance in variable-speed wind turbines. IEEE Trans. Sustain. Energy 2013, 4, 977-984. [CrossRef]

4. Chattot, J.-J. Extension of a helicoidal vortex model to account for blade flexibility and tower interference. J. Sol. Energy Eng. 2006, 128, 455-460. [CrossRef]

5. Gómez-Iradi, S.; Steijl, R.; Barakos, G.N. Development and validation of a CFD technique for the aerodynamic analysis of HAWT. J. Sol. Energy Eng. 2009, 131, 031009. [CrossRef]

6. Kim, H.; Lee, S.; Lee, S. Influence of blade-tower interaction in upwind-type horizontal axis wind turbines on aerodynamics. J. Mech. Sci. Technol. 2011, 25, 1351-1360. [CrossRef]

7. Hsu, M.-C.; Bazilevs, Y. Fluid-structure interaction modeling of wind turbines: Simulating the full machine. Comput. Mech. 2012, 50, 821-833. [CrossRef]

8. Li, Y.; Paik, K.-J.; Xing, T.; Carrica, P.M. Dynamic overset CFD simulations of wind turbine aerodynamics. Renew. Energy 2012, 37, 285-298. [CrossRef]

9. Wang, Q.; Zhou, H.; Wan, D. Numerical simulation of wind turbine blade-tower interaction. J. Mar. Sci. Appl. 2012, 11, 321-327. [CrossRef]

10. Hsu, M.-C.; Akkerman, I.; Bazilevs, Y. Finite element simulation of wind turbine aerodynamics: Validation study using NREL Phase VI experiment. Wind Energy 2014, 17, 461-481. [CrossRef]

11. Quallen, S.M. Development and Verification of a CFD Based Simulation Software for Floating Offshore. Ph.D. Thesis, University of Idaho, Moscow, ID, USA, 2015.

12. Duque, E.P.N.; van Dam, C.P.; Hughes, S.C. Navier-Stokes simulations of the NREL combined experiment Phase II rotor. In Proceedings of the 37th AIAA Aerospace Sciences Meeting and Exhibit, Reno, NV, USA, 11-14 January 1999; pp. 143-153.

13. Zahle, F.; Sørensen, N.N.; Johansen, J. Wind turbine rotor-tower interaction using an incompressible overset grid method. Wind Energy 2009, 12, 594-619. [CrossRef]

14. Shur, M.L.; Spalart, P.R.; Strelets, M.K.; Travin, A.K. A hybrid RANS-LES approach with delayed-DES and wall-modelled LES capabilities. Int. J. Heat Fluid Flow 2008, 29, 1638-1649. [CrossRef]

15. ANSYS, Inc. ANSYS Fluent Theory Guide, Release 17.1; ANSYS, Inc.: Canonsburg, PA, USA, 2016.

16. ANSYS, Inc. ANSYS Fluent User Guide, Release 17.1; ANSYS, Inc.: Canonsburg, PA, USA, 2016.

17. Eriksen, P.A.; Krogstad, P.Å. Experimental results for the NOWITECH/NORCOWE blindtest. Energy Procedia 2012, 24, 378-384. [CrossRef]

18. Krogstad, P.Å.; Eriksen, P.A. "Blind test" calculations of the performance and wake development for a model wind turbine. Renew. Energy 2013, 50, 325-333. [CrossRef]

19. Somers, D.M. The S825 and S826 Airfoils; Subcontractor Report NREL/SP-500-36344; National Renewable Energy Laboratory: Golden, CO, USA, 2005. 
20. Spalart, P.R.; Jou, W.H.; Strelets, M.; Allmaras, S.R. Comments on the feasibility of LES for wings, and on a hybrid RANS/LES approach. In Proceedings of the First AFOSR International Conference on DNS/LES, Ruston, LA, USA, 4-8 August 1997.

21. Wilcox, D.C. Turbulence Modeling for CFD, 2nd ed.; DCW Industries, Inc.: La Canada, CA, USA, 1998; pp. 185-201.

22. Menter, F.R. Two-equation eddy-viscosity turbulence models for engineering applications. AIAA J. 1994, 32, 1598-1605. [CrossRef]

23. Krogstad, P.A.; Lund, J.A. An experimental and numerical study of the performance of a model turbine. Wind Energy 2012, 15, 443-457. [CrossRef]

24. Luo, D.; Yan, C.; Liu, H.; Zhao, R. Comparative assessment of PANS and DES for simulation of flow past a circular cylinder. J. Wind Eng. Ind. Aerodyn. 2014, 134, 65-77. [CrossRef]

(C) 2017 by the authors; licensee MDPI, Basel, Switzerland. This article is an open access article distributed under the terms and conditions of the Creative Commons Attribution (CC BY) license (http:/ / creativecommons.org/licenses/by/4.0/). 